\title{
Polymer microparticle arrays from electrodynamically focused microdroplet streams
}

\author{
K. C. Ng, ${ }^{\text {a) }}$ J. V. Ford, S. C. Jacobson, J. M. Ramsey, and M. D. Barnes ${ }^{\text {b) }}$ \\ Chemical and Analytical Sciences Division, Oak Ridge National Laboratory, Oak Ridge, \\ Tennessee 37831-6142
}

(Received 12 January 2000; accepted for publication 9 February 2000)

\begin{abstract}
We describe instrumentation for forming two-dimensional arrays of polymer microparticles produced from electrodynamically focused microdroplet streams. A single-stage linear quadrupole was used to focus droplets/particles onto silanated glass slides mounted on a computer-driven two-dimensional translation stage. Center-to-center position stability was on the order of $1 \mu \mathrm{m}$. Applications to molecular sorting with polymer particles as host carriers are discussed. (c) 2000 American Institute of Physics. [S0034-6748(00)01506-9]
\end{abstract}

\section{INTRODUCTION}

In this article, we describe instrumentation and methodology for producing high-density ordered two-dimensional arrays of polymer or polymer-composite microparticles. Using a combination of microdroplet techniques and electrodynamic focusing, we are able to form dry polymer microspheres from droplets of solution and focus them with a linear quadrupole to a well-defined array location. A computer-controlled $X-Y$ translation stage was used to produce rectangular arrays of particles of varying size (6-15 $\mu \mathrm{m}$ nominal diameter) with interparticle distances of 25-50 $\mu \mathrm{m}$ (typically 3-4 particle diameters). We describe some of the issues and limitations associated with this experimental approach as well as potential applications to systematic (high-speed) microparticle study, ${ }^{1-4}$ single-molecule imaging and spectroscopy, ${ }^{5,6}$ isolation of fluorescently labeled polymer particles from solution, ${ }^{7}$ and the study of polymer microcomposites. ${ }^{8,9}$

While the production of microarrays via "ink-jet" deposition of droplets of solution is fairly common for use in biochemical assays for example, there is very little literature on the production of ordered arrays of dry polymer particles. To our knowledge, this is the first report of production of polymer microparticle arrays from solution using electrodynamic focusing. Mesoscale particle manipulation using different techniques is now attracting some attention for a variety of applications. Renn and co-workers have recently demonstrated laser guidance of microparticles in hollow-core optical fibers, ${ }^{10,11}$ and Davis and co-workers have examined drying behavior and electrophoresis ${ }^{12}$ of microparticles entrained in a laminar gas flow ${ }^{13}$ and particle manipulation using electrodynamic ${ }^{14}$ and optical levitation approaches. ${ }^{15}$

\section{EXPERIMENT}

Arrays of polymer microparticles were produced using instrumentation similar to that developed for probing single

\footnotetext{
${ }^{a}$ Permanent address: Department of Chemistry, California State University, Fresno, CA 93740.

b) Author to whom correspondence should be addressed.
}

fluorescent molecules in electrodynamically focused droplet streams. ${ }^{16,17}$ Figure 1 shows a schematic of the apparatus used. Briefly, liquid microdroplets (30-40 $\mu \mathrm{m}$ diameter) of dilute polymer solution $(\leqslant 1 \% \mathrm{w} / \mathrm{w}$ polymer) were injected into a linear quadrupole. An ac voltage of 200-600 V (peak to peak) and $200-800 \mathrm{~Hz}$ was applied to the (10-cm-long) 1/16-in-diam stainless steel rods with a 0.2 in. center-tocenter spacing (a relative phase difference of $180^{\circ}$ on each adjacent rod). A combination of low-voltage function generator (Stanford Research DS335), a power amplifier (Kepco BOP $1000 \mathrm{M}$ ), and $5 \times$ step-up transformer were used to produce the focusing voltages. The long rod configuration was used to ensure that nearly all the solvent evaporated from the droplet en route to the microscope slide.

We found that chemical treatment of the standard glass microscope slides was essential for ordered particle array generation. Without pretreatment with a silanating agent, the particle positions were observed to be totally randomized, presumably due to the presence of stray charges on the surface of the glass. This problem was solved using a straightforward silanation procedure to chemically modify the surface of the slide. After cleaning in an ultrasonic bath, the slides were etched briefly $(\approx 60 \mathrm{~s}$ ) in buffered oxide etchant (BOE, a mixture of $\mathrm{HF}$ and $\mathrm{NH}_{4} \mathrm{~F}$ ), and suspended above the vapor of a heated hexamethyl disilazane (HDMS) silanating agent (MicroPrime) for about $30 \mathrm{~min}$.

The slides were mounted kinematically on a computercontrolled $X-Y$ translation stage (Parker-Daedal Model 0815987) driven in discrete steps of 25-100 $\mu \mathrm{m}$ with $0.1 \mu \mathrm{m}$ precision. The motion of the stage was synchronized with the droplet stream using a TTL-synch output from the droplet generator driver coupled with a digital delay generator (Stanford Research DG535). Figure 2 shows a differential interference contrast (DIC) micrograph of $9.5 \mu \mathrm{m}$ polyethylene glycol (10k MW) polymer particles produced from $45 \mu \mathrm{m}$ (nom.) liquid droplets deposited on a silanated glass slide. As indicated in Fig. 2, the measured center-to-center particle spacing was $38.5 \mu \mathrm{m}$. Bright-field micrographs show particle images that are consistent with spherical transparent particles under bright-field illumination, that is, a bright central spot 


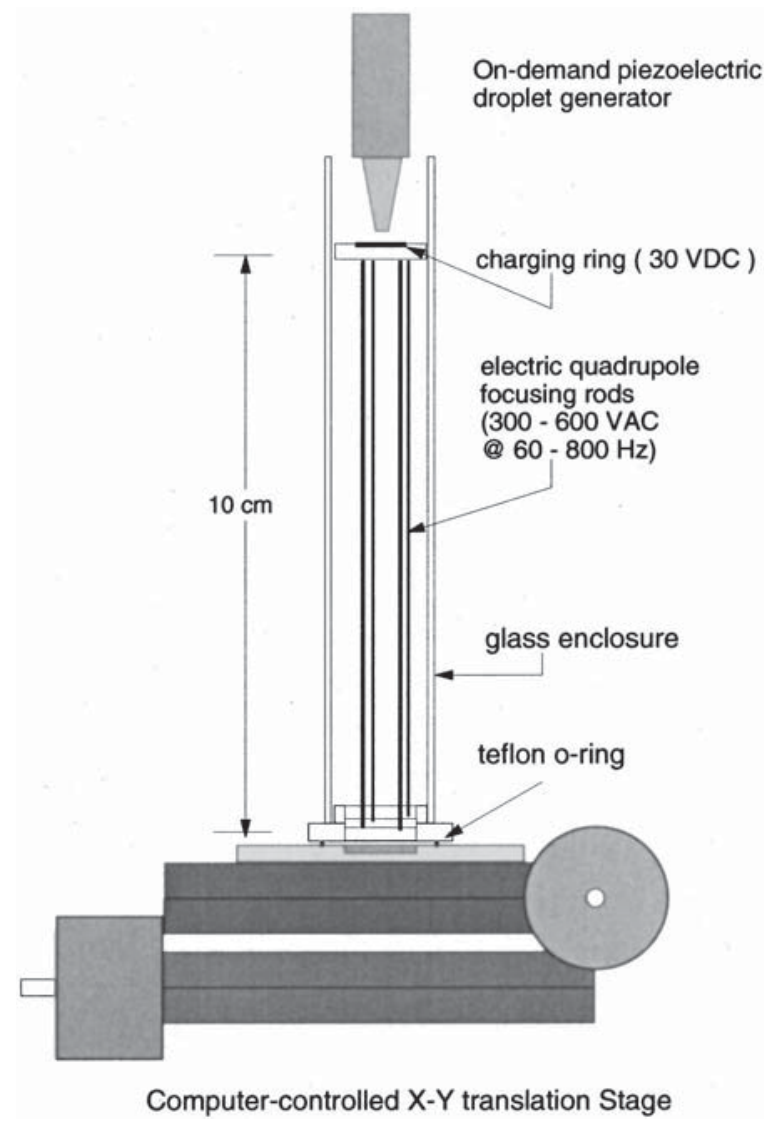

FIG. 1. Schematic of apparatus for generating 2D arrays of polymer particles from electrodynamically focused microdroplet streams.

surrounded by a dark circular background due to refraction of light near the edges of the sphere. The differential interference contrast images (DIC) show more clearly the morphology of the particles that are observed to be highly spherical. Similar results were obtained for $7 \mu \mathrm{m}$ PEG particles with a $30 \mu \mathrm{m}$ center-to-center spacing, where the (dry) particle size was reduced by dilution of the PEG solution.

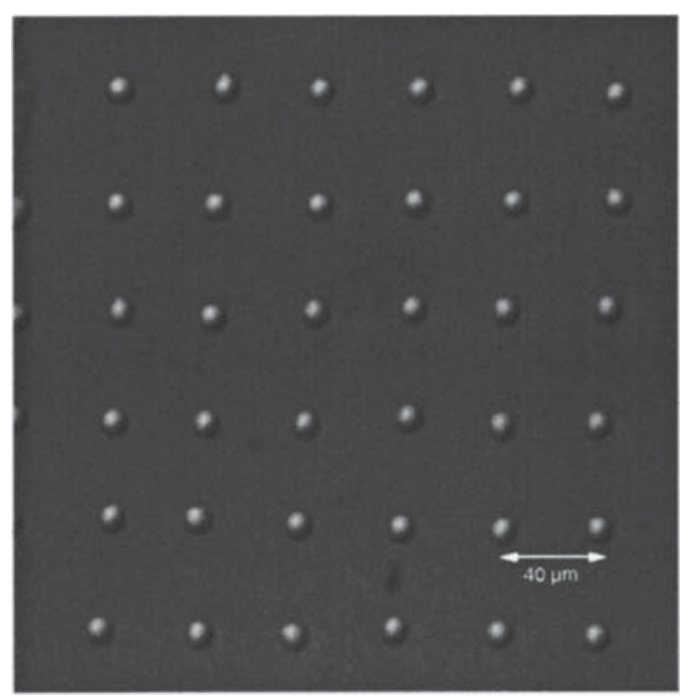

FIG. 2. Differential interference contrast (DIC) micrograph of a segment of $9.5 \mu \mathrm{m}$ polyethylene glycol particle array produced using a linear quadrupole. The interparticle spacing was $38.5 \mu \mathrm{m}$.
For reasons that are not yet clear, attempts to further decrease the center-to-center particle spacing resulted in particle agglomeration. This is curious since the particles all bear the same sign charge and the interparticle distance is enormous on the length scale of a van der Waals interaction.

We analyzed the center-to-center spacing statistics to obtain an estimate of positioning resolution in our configuration. For the data shown in Fig. 2, the average horizontal and vertical particle spacing and standard deviations were $\langle\Delta x\rangle=37.8 \mu \mathrm{m} \pm 1.7 \mu \mathrm{m},\langle\Delta y\rangle=38.2 \mu \mathrm{m} \pm 1.8 \mu \mathrm{m}$, respectively. We also observed some systematic column registration errors due to nonuniform timing. Since the terminal particle velocity is proportional to the square of the diameter, small size fluctuations may result in differences in particle velocity that in turn can produce registration errors as a new row is formed. We anticipate that this problem can be solved in a straightforward way by triggering the motion of the translation stage using an elastic scattering pulse from a photodiode. The standard deviations in horizontal and vertical spacing are similar and much larger than the $\pm 0.1 \mu \mathrm{m}$ precision in stage motion. The dominant error comes from Brownian fluctuations in radial position whose rms value increases as the particle size decreases. For 10- $\mu \mathrm{m}$-diam particles, we had estimated peak-to-peak radial position fluctuations of approximately $1 \mu \mathrm{m}$ on the basis of trajectory imaging. There are likely additional trajectory perturbations as the particles leave the quadrupole field along the $\approx 5 \mathrm{~mm}$ transit distance from the exit plane of the quadrupole to the glass slide.

There are a number of interesting and useful applications of the quadrupole-guided particle array generation apparatus that we have described here. In particular, there has been recent interest in polymer-composite and polymer alloy micro- and nanoparticles and a need for correlating dynamic properties probed "on-the-fly" with static morphological properties of dried particles. ${ }^{2,8,18}$ The instrumentation described here provides a convenient way of establishing a registration or correspondence between a particle probed upstream and an array address. In addition, the experimental layout is convenient for a number of particle-sorting and switching applications. Since the particles are all electrically charged, it is straightforward to "reject" or switch out particles that fail to meet a sort criterion using a pulsed voltage on a switching electrode. Simple examples of such sort criteria are upstream fluorescence (amplitude or color) or other elastic light-scattering probes. One can sort particles containing single molecules from "empty" particles, or sort on the basis of morphological characteristics such as material homogeneity, or particles that satisfy optical resonance conditions. Finally, the electric quadrupole can be used as a charge-to-mass filter for polymer nano- and mesoparticles (up to 1 or $2 \mu \mathrm{m}$ in size) to selectively collect particles of a preset size with very high size discrimination.

\section{ACKNOWLEDGMENTS}

This research was sponsored by Office of Basic Energy Sciences, Divisions of Chemical and Material Sciences, U.S. Department of Energy, under Contract No. DE-AC05- 
960R22464 with Oak Ridge National Laboratory, managed by Lockheed Martin Energy Research Corporation. K. C. Ng acknowledges support from the ORNL Faculty Research Participation Program J. V. Ford acknowledges support from the ORNL Postdoctoral Research Program.

${ }^{1}$ K. Fukui, B. G. Sumpter, K. Runge, M. D. Barnes, and D. W. Noid, Chem. Phys. 244, 339 (1999).

${ }^{2}$ M. D. Barnes, K. C. Ng, and K. Fukui, B. G. Sumpter, and D. W. Noid, Materials Today 2, 25 (1999).

${ }^{3}$ S. Arnold, S. Holler, and N. L. Goddard, Mater. Sci. Eng., B 48, 139 (1997).

${ }^{4}$ M. D. Barnes, C-Y. Kung, W. B. Whitten, J. M. Ramsey, S. Arnold, and S. Holler, Phys. Rev. Lett. 76, 3931 (1996).

${ }^{5}$ M. D. Barnes, K. C. Ng, K. P. McNamara, C-Y. Kung, J. M. Ramsey, and S. C. Hill, Cytometry 36, 169 (1999).

${ }^{6}$ S. C. Hill, P. Nachman, S. Arnold, J. M. Ramsey, and M. D. Barnes, J. Opt. Soc. Am. B 16, 1868 (1999).

${ }^{7}$ W. B. Whitten, M. J. Shapiro, J. M. Ramsey, and B. V. Bronk, Appl. Opt. 34, 3203 (1995).
${ }^{8}$ M. D. Barnes, C-Y. Kung, N. Lermer, K. Fukui, B. G. Sumpter, D. W. Noid, and J. U. Otaigbe, Opt. Lett. 24, 121 (1999).

${ }^{9}$ M. D. Barnes, K. C. Ng, K. Fukui, B. G. Sumpter, and D. W. Noid, Macromolecules 32, 7183 (1999).

${ }^{10}$ M. J. Renn, R. Pastel, and H. J. Lewandowski, Phys. Rev. Lett. 82, 1574 (1999).

${ }^{11}$ M. J. Renn and R. Pastel, J. Vac. Sci. Technol. B 16, 3859 (1998).

${ }^{12}$ T. J. Johnson and E. J. Davis, J. Colloid Interface Sci. 215, 397 (1999).

${ }^{13}$ R. Vehring, C. L. Aardahl, G. Schweiger, and E. J. Davis, J. Aerosol Sci. 29, 1045 (1998).

${ }^{14}$ R. Vehring, C. L. Aardahl, E. J. Davis, G. Schweiger, and D. S. Covert, Rev. Sci. Instrum. 68, 70 (1997).

${ }^{15}$ A. Ashkin, Phys. Rev. Lett. 24, 156 (1970).

${ }^{16}$ M. D. Barnes, N. Lermer, C-Y. Kung, W. B. Whitten, and J. M. Ramsey, Opt. Lett. 22, 1265 (1997).

${ }^{17}$ N. Lermer, M. D. Barnes, C-Y. Kung, W. B. Whitten, and J. M. Ramsey, Anal. Chem. 69, 2115 (1997).

${ }^{18}$ S. Holler, M. Surbek, R. K. Chang, and Y. L. Pan, Opt. Lett. 24, 1185 (1999). 